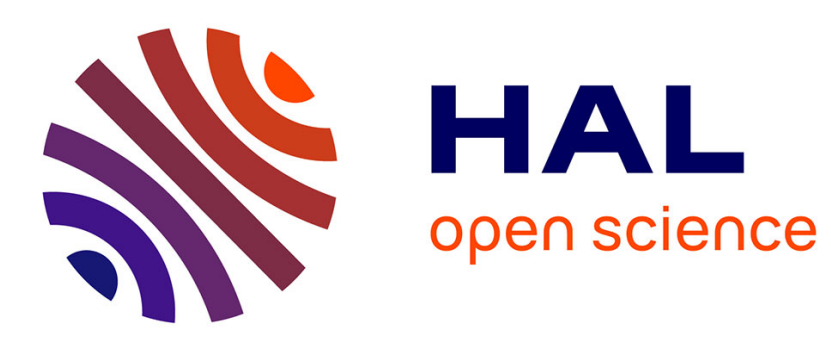

\title{
Wolbachia prevalence, diversity, and ability to induce cytoplasmic incompatibility in mosquitoes
}

\author{
Mathieu Sicard, Manon Bonneau, Mylene Weill
}

\section{To cite this version:}

Mathieu Sicard, Manon Bonneau, Mylene Weill. Wolbachia prevalence, diversity, and ability to induce cytoplasmic incompatibility in mosquitoes. Current Opinion in Insect Science, 2019, 34, pp.12-20. 10.1016/j.cois.2019.02.005 . hal-02114344

\section{HAL Id: hal-02114344 \\ https://hal.science/hal-02114344}

Submitted on 18 Nov 2020

HAL is a multi-disciplinary open access archive for the deposit and dissemination of scientific research documents, whether they are published or not. The documents may come from teaching and research institutions in France or abroad, or from public or private research centers.
L'archive ouverte pluridisciplinaire HAL, est destinée au dépôt et à la diffusion de documents scientifiques de niveau recherche, publiés ou non, émanant des établissements d'enseignement et de recherche français ou étrangers, des laboratoires publics ou privés. 


\section{Wolbachia prevalence, diversity, and ability to induce cytoplasmic incompatibility in mosquitoes Mathieu Sicard, Manon Bonneau and Mylene Weill}

\begin{abstract}
To protect humans and domestic animals from mosquito borne diseases, alternative methods to chemical insecticides have to befound. Pilot studies using the vertically transmitted bacterial endosymbiont Wolbachiawere already launched in different parts of the world. Wolbachia can be used either in Incompatible Insect Technique (IIT), to decrease mosquito population, or to decrease the ability of mosquitoes to transmit pathogens. Notall mosquito species are naturally infected with Wolbachia: while in Culex pipiens and Aedes albopictus almost all individuals harbor Wolbachia, putative infections have to be further investigated in Anopheles species and in Aedes aegypti. All Wolbachia-based control methods rely on the ability of Wolbachia to induce cytoplasmic incompatibility $(\mathrm{Cl})$ resulting in embryonic death in incompatible crossings. Knowledge on $\mathrm{Cl}$ diversity in mosquito is required to find the better Wolbachia-mosquito associations to optimize the success of both 'sterile insect' and 'pathogen blocking' Wolbachia-based methods.
\end{abstract}

\section{Address}

ISEM, University of Montpellier, CNRS, EPHE, IRD, Montpellier, France

Corresponding authors:

Sicard, Mathieu (mathieu.sicard@umontpellier.fr), Weill, Myle ne (mylene.weill@umontpellier.fr)

\section{Current Opinion in Insect Science 2019, 34:xx-yy}

This review comes from a themed issue on Vectors and medical and veterinary entomology

Edited by Anna Cohuet and Claudio Lazzari

\section{Introduction}

Mosquitoes are vectors for major pathogens such as arboviruses, nematodes and protozoans. To protect humans and domestic animals from these pathogens, strategies targeting the vectors aim at decreasing vector population density and/or at diminishing their ability to transmit pathogens $\left[1^{\circ}{ }^{\circ}\right]$. Presently, the most common vector control actions are intended to decrease the longevity and the density in vector populations, mainly by using chemical insecticides, which have reached their limits because of genetic resistance and negative consequence on non-targeted invertebrate species [2]. In this context, new vector control strategies, hopefully more environmental friendly, have to be proposed. Biocontrol strategies of vectors based on the knowledge of their microbiota are promising [3], and particularly those based on $a$-proteobacteria of the genus Wolbachia which manipulate many aspects of their mosquito host biology [4-6]. Because these symbionts can influence both mosquito reproduction and their pathogen loads, Wolbachiabased control methods can be deployed to reduce vector populations and/or to diminish their capacity to transmit pathogens.

The Wolbachia, which are maternally transmitted endosymbionts, have long been studied because of their ability to manipulate their host reproduction to increase their prevalence within host populations [4]. Cytoplasmic incompatibility (CI) is the mostfrequent manipulation used by Wolbachia to spread within insect populations [4]. During the invasion process, CIoccurs when malesinfected with a given Wolbachia breed with uninfected females which, then, produce non-viable embryos (Figure 1). As crossings between individualsinfected with compatible Wolbachia give normal viableembryos, the consequence of CI is that the prevalence of Wolbachia increases within the host population so that prevalence can reach $100 \%$.

The ability of Wolbachia to induce CI is the cornerstone of the two major Wolbachia-based control methods developed to date (Box 1). The first method, called 'Incompatible Insect Techniques' (IIT), is related to the classical sterile insecttechnics (SIT) $\left[1^{\bullet \bullet}\right.$, andaims atdecreasingmosquito population size by releasing Wolbachia infected males that are incompatible with local females. In this strategy, the localfemales producenon-viable embryos resulting locally and temporally in the vector population crash-down $\left[7,8,9^{\bullet \bullet}, 10\right]$. The second method uses CI induction by Wolbachia, not to reduce the density of a focal vector population, but to sustainably replace its uninfected individuals by Wolbachia infected ones. Indeed it has been shown that Wolbachia can interfere negatively with the transmission of disease pathogens including the major arboviruses Chikungunya, Dengue, Rift Valley, West-Nile, Zika, and so on $\left[11^{\bullet}, 12^{\bullet \bullet}, 13^{\bullet \bullet}, 14-18,19^{\circ}\right]$. In this strategy, CIallows theprogressive invasionoflocalvector population with individuals harboring Wolbachia which mediate blocking of arboviruses transmission [20-22].

Wolbachia being a promising weapon against mosquitoes, we synthetize in this review the current knowledge 


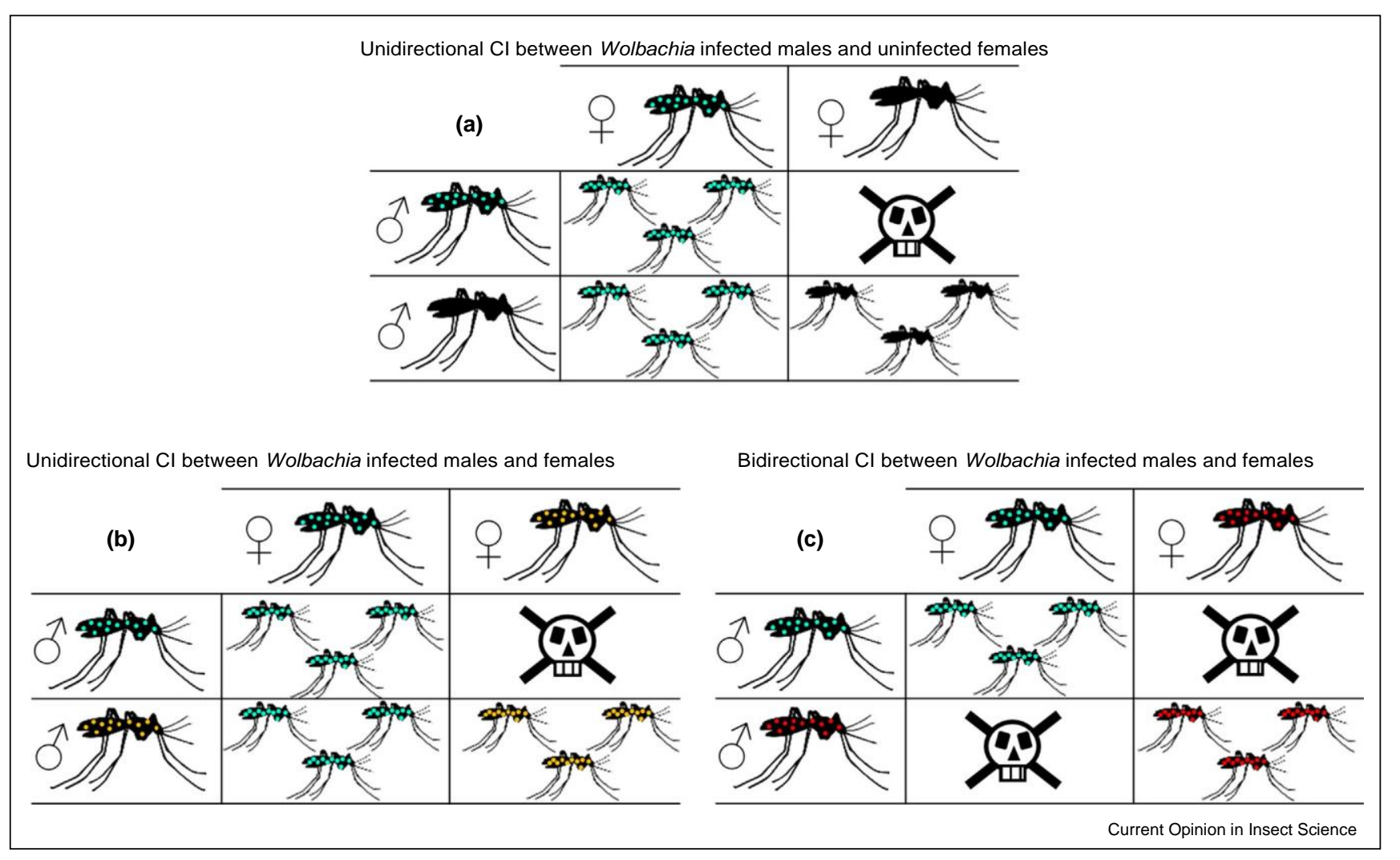

The different types of $\mathrm{Cl}$ in mosquitoes.

(a) The simplest type of $\mathrm{Cl}$ occurring between Wolbachia infected males and uninfected females allows Wolbachia to invade uninfected populations. In Culex pipiens and Aedes albopictus, Wolbachia has reached fixation in nature; this type of Cl is, thus, only observed in laboratory conditions when females are artificially cured of their Wolbachia with an antibiotic treatment. This type of Cl was also observed when Ae. aegypti and An. stephensimales were transinfected with a Wolbachia strain from other species and crossed with naturally uninfected females. (b-c) Other types of $\mathrm{Cl}$ can occur between males and females both infected with different Wolbachia strains. In such crosses $\mathrm{Cl}$ can be unidirectional (i.e. only one of the reciprocal crosses is compatible, the other is incompatible) (b) or bidirectional (both reciprocal crosses are incompatible) (c). Unidirectional CI can be observed in Ae. albopictus when bi-infected males (infected with both wAlbA and wAlbB) are crossed with laboratory females infected only with wAlbA. In Culexpipiens, really complex $\mathrm{Cl}$ crossing types are observed, including unidirectional and bidirectional $\mathrm{Cl}$ depending on the wPip strains present in crossed individuals.

accumulated on Wolbachia prevalence, diversity, and ability to induce CIin the major vector mosquito species of Aedes, Anopheles, and Culex genera.

\section{Prevalence and diversity of Wolbachia in mosquitoes}

The diversity hidden behind the term 'Wolbachia pipientis' in arthropod and nematode hosts is presently organized in 17 phylogenetic clades called supergroups (A to Q) [23-27]. Within each supergroup, the unit of diversity is called a 'strain'. Most of the Wolbachia strains were named according to their host species (e.g. $w$ Pipin Culex pipiens and wAlb in Aedes albopictus). If genetic differences are identified within an already defined 'strain', new information including sampling location or phylogenetic position can be added to name the new strains. The increase in genomic analyses to investigate more and more accurately Wolbachia diversity, between and within host species, may lead to important changes in the definition of new 'strains' in the near future [28].

Anopheles mosquitoes, the major vectors of Plasmodium, were considered to be exempt of Wolbachia because classic PCR diagnostic tests were always found negative [29-31]. However, very deep sequencing of Wolbachiaspecific 16S rRNA recently suggested putative natural infections of Anopheles coluzzii and Anopheles gambiae in Burkina Faso [32 $\left.{ }^{\bullet}, 33\right]$. The Wolbachia $16 \mathrm{~S}$ sequences obtained were attributed to a new strain named ' $w$ Anga'. Positive mothers did not produce only positive offspring ruling out both an insertion in host genome and a perfect vertical transmission of Wolbachia [32 $2^{\circ}$. Such a genetic detection of Wolbachia has now been extended to 
Box 1 Wolbachia anti-vectorial methods: either decrease the density or modify the physiology of mosquitoes

a In the Incompatible Insect Technique (IIT) large numbers of Wolbachia-infected males are released. The Wolbachia harboring by these males has to be carefully chosen to ensure that these males will be able to kill, due to $\mathrm{Cl}$, embryos of females from the focal population. This requires that females either do not harbor the same Wolbachia strain (for instance in Ae. albopictus and C. pipiens cases) or that are putatively not infected with Wolbachia (for instance in Ae. aegypti and An. gambiae). After repeated releases of incompatible males, the vector population will decrease. To be successful, the Wolbachia strain in the released males should be involved in bidirectional Cl with the Wolbachia strain from the targeted population (Figures 1 and 2). This way, the released Wolbachia has almost no chance to settle in the introduced environment because even if females are concomitantly released with infected males, they would not be able to produce offspring with the local males. The release of the same line of Wolbachia infected males can stay efficient through years. For even more efficiency, ITT may be combined with sterile insect technique (SIT) by irradiating Wolbachia-infected mosquitoes.

b Wolbachia can also be used to modify the physiology of mosquito. In this method, both Wolbachia infected male and female mosquitoes are released. Cl allows the progressive invasion of local vector population with individuals harboring Wolbachia which mediates blocking of arboviruses transmission. To be successful, the Wolbachia of the released individuals have (i) to block viruses and (ii) to exhibit a unidirectional $\mathrm{Cl}$ relationship with the targeted populations that allows the spread and sustainability of protective Wolbachia (Figure 2).

Anophelesfunestusfrom Senegal [34], Anopheles arabiensis in Tanzania [35], 16 Anopheles species among a total of 25 in Gabon [36], and in 5 species among 17 from Ghana, Democratic Republic of the Congo (DRC), Guinea, Uganda and Madagascar [37]. Wolbachia 16S sequences exhibited much larger diversity than usually expected within one strain suggesting multiple infections. Indeed, these sequences can be clustered with those from $\mathrm{Wol}$ bachia strains belonging to the supergroups A, Band even, more surprisingly, to supergroup $\mathrm{C}\left[36,38^{\bullet \bullet}\right]$. The reported proportions of positive individuals vary among species and localities but remain low for most of the Anopheles species. Besides theinterestingcase of Anopheles moucheti in Gabon and DRC, for which the prevalence of Wolbachia seems very high, calls for further studies $[36,37]$. The low detection of Wolbachia in most Anopheles species could be duetoalow prevalence of the symbiont that required more individuals and screened populations to be detected. However, if Wolbachia are really present in these Anopheles species their density must be very low as several screening technics revealed discordant results, even requiring nested PCR or quantitative PCR with a very high number of amplification cycles for detection [34]. The main problem is that the putative presence of Wolbachia in all these Anopheles species is mostly based on its genetic detection which is not an actual proof of real infection and could result from contaminations, at least for certain species [ $38^{\bullet \bullet}$. To our knowledge, no electronic microscopy observations that would provide a direct proof of infections, have been yet conducted. Only one study reported fluorescent in situ hybridization (FISH) labelling to monitor the presence of Wolbachia, found at low density in the ovaries of some An. coluzzii [33].

Aedes aegypti and Ae. albopictus, the major arboviruses vectors, although belonging to the same genus exhibit strongly different patterns in terms of Wolbachia infection. As for Anopheles, classic PCR tests were always negative on Ae. aegypti placing this species among uninfected ones. However, deep sequencing of Wolbachia-specific 16S rRNA from both larvae and adults in USA and Thailand were recently found positive, indicating the putative presence of Wolbachiain some individuals [39,40]. $\mathrm{Nev}^{-}$ ertheless, as it is the case with the Anopheles, if Wolbachia cells are present in this vector it must be at low prevalence and at a 'cryptic' load [41]. Further investigations including symbiont visualization must be conducted in the future to confirm the presence of Wolbachia at low prevalence and titer in Ae. aegypti.

In contrast, Ae. albopictus is found infected with Wolbachia everywhere in the world [42,43]. All individuals are usually infected with two Wolbachia strains namely $w \mathrm{AlbA}$ and $w \mathrm{AlbB}$ belonging to the supergroups A and B, respectively [44]. However, a polymorphism of the infection status exists: (i) $w$ AlbB mono-infected males (but not females) have been reported in La Réunion Island and Madagascar field populations [45], and (ii) $w A l b A$ mono-infected laboratory lines were obtained from individuals initially sampled in Thailand and Mauritius $[46,47]$. The genetic variation within both $w \mathrm{AlbA}$ and $w \mathrm{AlbB}$ strains is yet considered to be low as no variation was detected within each strain based on 16S rRNA, wsp and ftsZ gene sequences [42,46-48] suggesting that Wolbachia could have recently, invaded and spread throughout populations of this mosquito species to finally reach fixation [42].

In C. pipiens (s.l.), all individuals are infected with $w$ Pip Wolbachia that were also found non-polymorphic using MLST genes [23,24]. However, MLSTs including a larger number of highly polymorphic genes (MutL, ank2, pk1, pk2, GP12, GP15, and RepA) allowed to uncover a previously hidden diversity [49]. All $w$ Pip strains are monophyletic and closely related, and they form five groups from $w$ Pip-I to $w$ Pip-V. As the thousands C. pipiens individuals tested around the world [50-53] harbored a $w$ Pip strain belonging to one of the five groups, the infection is considered to have reached fixation in this species. However, few individuals in South Africa, France, Scotland and Tunisia were found negative to Wolbachia genetic tests [54,55]. A phylogenetic analysis based on mitochondrial markers demonstrated that all these uninfected mosquitoes form a new species named " 
Culex juppinov. sp.'independent from all the infected $C$. pipiens [55].

\section{$\mathrm{Cl}$ induction in natural Wolbachia-mosquito associations}

In Anopheles no cytoplasmic incompatibility has been shown in laboratory crosses between males putatively infected with Wolbachia and uninfected females [33]. Such laboratory observations are in accordance with the low detection of the symbionts in Anopheles natural populations. However, an acceleration of egg laying in Wolbachia positive females has been reported [33]. In Ae. albopictus, both $w \mathrm{AlbA}$ and $w \mathrm{AlbB}$ were reported to increase host fecundity [8]. CI does not occur between individuals from lines originating from distant parts of the world since most individuals are bi-infected with $w \mathrm{AlbA}$ and $w \mathrm{AlbB}$ showing no or low polymorphism [43] (Figure 1). Consequently, there is only one dominant crossing type in Ae. albopictus natural populations all over the world, resulting in compatibility between all lines. Nevertheless, females mono-infected with only $w$ AlbA strain produce unviable embryos when crossed with normally bi-infected males resulting in unidirectional CI [46,47] (Figure 1). This clearly demonstrated that $w \mathrm{AlbB}$ strain is able to induce CI but that this CI phenotype rarely occurs in nature because of the high frequency of bi-infections with $w \mathrm{AlbA}$ and $w \mathrm{AlbB}$.

In contrast to the absence of CI recorded in Anopheles and the poor crossing type diversity observed in Ae. albopictus, the hundreds of crosses performed between C. pipiens lines sampled worldwide have revealed an unrivaled diversity of crossing types $\left[56,57^{\circ}, 58\right]$ (Figure 1). Genetic diversity within the $w$ Pip clade is responsible for this unique CI polymorphism since (i) no other manipulative endosymbiont was detected in this host species, (ii) the host genetic background did not influence the crossing types, and more importantly (iii) C. pipiens lines harboring $w$ Pip belonging to the same phylogenetic group ( $w$ Pip-IV) are generally compatible, whereas 'inter-group crosses' are more likely to be incompatible [ $\left.57^{\circ}\right]$. Infected males harboring a $w$ Pip strain (from any $w$ Pip group) induce total CI(i.e. no embryo will develop) when crossed with uninfected females while the reciprocal crossing is fertile [59-61,62 ${ }^{\circ}$. Such unidirectional CI pattern between uninfected and infected individuals has certainly prevailed during the spread of the $w$ Pip infection in C.pipiens populations, but is no longer observed in the wild since infection reached fixation. To date, crosses can only occur between (i) individuals infected with the same $w$ Pip group (usually resulting in normal reproduction) or (ii) individuals harboring $w$ Pip from two different groups. Such 'inter-group crossings' can have three outcomes (Figure 1): (i) production of living offspring; (ii) unidirectional CI (one cross direction is compatible while the reciprocal one is incompatible) or (iii) bidirectional CI (both cross directions are incompatible).

\section{$\mathrm{Cl}$ induction in artificial Wolbachia-mosquito associations}

When Wolbachia have been experimentally introduced by transinfection in two "non-infected mosquito species' namely Ae. aegypti and Anopheles stephensi, CI has been observed showing that Wolbachia molecular targets responsible for CI are present in these species. Indeed, Ae. aegypti has been successfully transinfected independently with eight Wolbachia strains ( $w \mathrm{Mel}$, $w \mathrm{MelPop}$-CLA, $w \mathrm{MelCS}, w \mathrm{Ri}, w \mathrm{Au}, w \mathrm{AlbA}, w \mathrm{AlbB}$, and $w$ Pip $\left[14,19^{\circ}, 63-65\right]$ ) (Figure 2 ); and all induced unidirectional CI with natural uninfected Ae. aegypti except $w$ Au which is a Wolbachia strain from $D$. simulans that also does not induce CI in its natural host [19 ${ }^{\circ}$. An. stephensi has also been successfully transinfected with $w \mathrm{AlbB}$ from Ae. albopictus which induced CI enabling Wolbachia to invade uninfected laboratory populations [66]. Transinfections have also been conducted in Ae. albopictus, which is naturally infected, in order to create new crossing types. Both $w$ Pip and $w \mathrm{Mel}$ strains have been introduced in Wolbachia-cured lines resulting in bidirectional incompatibility between transinfected lines and naturally infected ones [67-69]. Moreover, a triple-infected ( $w \mathrm{AlbA}, w \mathrm{AlbB}$, and $w \mathrm{Pip})$ Ae. albopictus line has been established; it expresses unidirectional CI when crossed with naturally double-infected mosquitoes.

C. pipiens has not yet been transinfected with other Wolbachia since the natural crossing type diversity demonstrated in this species can provide with unidirectional and bidirectional crossing types required in Wolbachiabased control methods (e.g. [53]).

\section{Cellular mechanism of $\mathrm{Cl}$ in mosquitoes}

The cellular mechanism of CI has only been yet studied in details in $C$. pipiens $\left[62^{\circ}\right]$. To do so, the early embryogenesis was monitored using fluorescence confocal microscopy in (i) fertile intra-group crosses,

(ii) incompatible crosses between infected males and infected females (i.e. inter-group crosses), and (iii) incompatible crosses between infected males and uninfected females. Despite the diversity of the crosses involving various $w$ Pip strains, common embryonic defects resulting in the death of the embryos were detected. These defects consisted in paternal chromatin condensation and segregation impairments during the first embryonic division as for Drosophila and Nasonia [62,70-74] (Figure 3).

Wolbachia genes involved in $\mathrm{Cl}$ in mosquitoes Cytological observations in C. pipiens suggest that a toxin, deposited in maturing sperm, would prevent the development of embryos by impairing paternal 


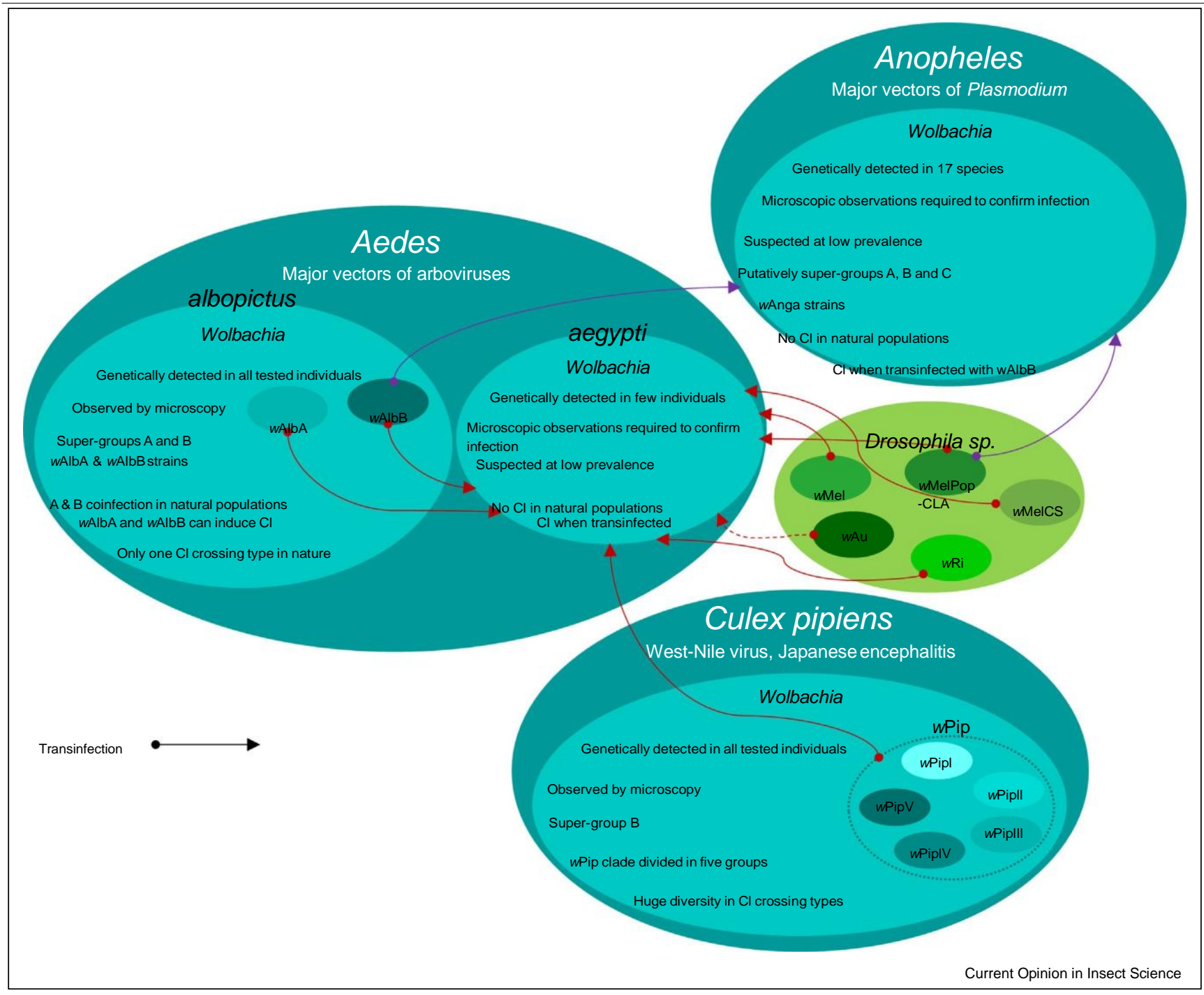

A synthetic view of Wolbachia knowledge in mosquitoes.

Arrows represent Wolbachia transinfection from one mosquito donor species to a recipient mosquito species or from Drosophila to a mosquito recipient species. The Anopheles transinfected species was An. stephensi[66]. Solid arrows represent strains that were able to induce $\mathrm{Cl}$ when transferred to a recipient host while the dashed arrow represent the strain wAu that does not induce $\mathrm{Cl}$.

chromatin normal segregation unless they are rescued by an antidote [75]. A combination of approaches on different insects demonstrated that the Wolbachia cidA and $\operatorname{cidB}$ genes, first identified by the presence of CidA protein in C.pipiens sperm [76], were the determinantin the induction and rescue of $\mathrm{CI}$ in insects $\left[77^{\bullet \bullet}, 78^{\bullet \bullet}\right]$. Biochemical analyses revealed that CidB protein could act as toxin since it encodes a putatively toxic deubiquitylase (DUB). Convincingly, when cidA and cidB were transgenically expressed in uninfected Drosophila males, these males were incompatible with uninfected females: embryos were unviable, and the first embryonic mitosis displayed the same characteristics as in CI $\left[77^{\bullet \bullet}, 78^{\bullet \bullet}\right.$. CidA is most probably the antidote against the toxic activity of CidB since its expression during early oogenesis restored the viability of uninfected eggs fertilized by Drosophila infected males [79]. Both cidA and $c i d B$ genes are monomorphic in $w \mathrm{AlbB}$. No genomic data are yet available on $w A l b A$ and on the putative strain wAnga from Anopheles. However, in C. pipiens, these genes are amplified and diversified within each ${ }_{w}$ Pip genome constituting the fuel for the diversity of crossing types described in this species [ $\left.80^{\circ}\right]$. This cidA/ $c i d B$ gene amplifications and diversifications in $w$ Pip may also account for the impressive CI penetrance observed in C. pipiens [ $62^{\circ}$ ]. 


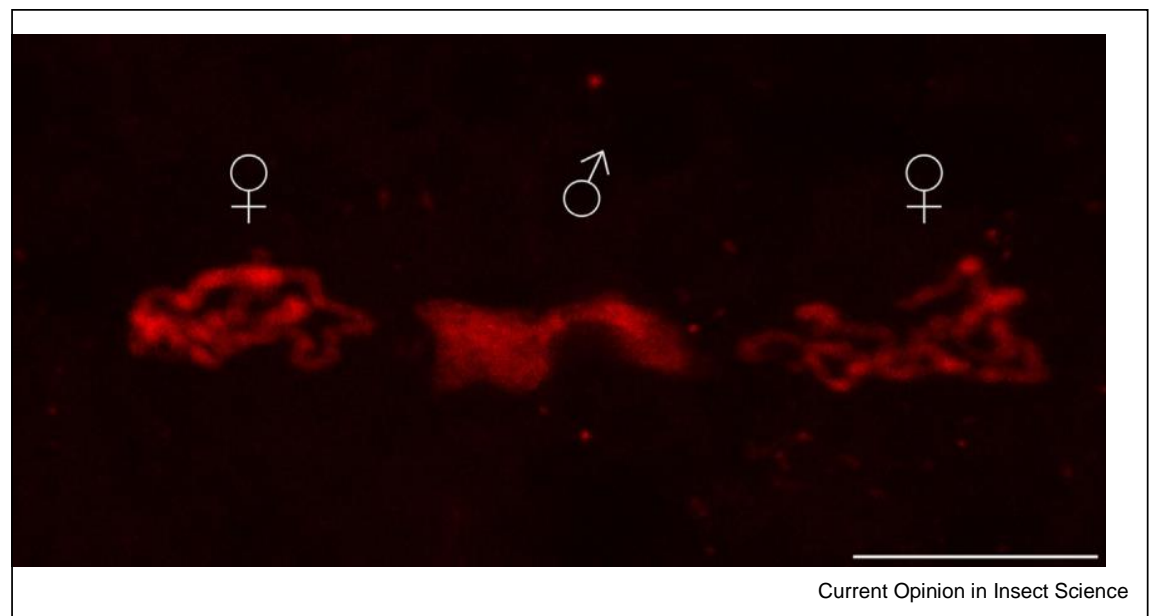

$\mathrm{Cl}$ cellular phenotype as observed after the first embryonic division in Culex pipiens.

Paternal chromatin and maternal chromatin were labelled using propidium iodide and were observed under confocal microscopy. In the picture, we can see that paternal chromatin $(<)$ failed to segregate during the first mitotic embryonic division while maternal chromatin (,) did segregate. Because of this defect in the first division, the embryos will not be able to develop normally into larvae. Scale bar is $10 \mathrm{~mm}$.

\section{Conclusion}

Prevalence and diversity of Wolbachia are quite contrasted between mosquito species. Ae. albopictus and C. pipiens individuals all harbor diverse Wolbachia that can induce CI and influence their life history traits at each generation. In contrast, the major arboviruses vector Ae. aegypti and the major malaria vectors, Anopheles spp., are only suspected to be infected. Further studies are required to investigate infection status of these last species. Recent studies on $C$. pipiens along with those conduced on Drosophila brought new elements on CI mechanisms, both at sellular and molecular levels that constitute the cornerstone for an efficient use of Wolbachia genetic resources in vector control.

\section{Acknowledgements}

This work was supported by French ANR [project 'CIAWOL' ANR-16CE02-0006-01] coordinated by Mathieu Sicard. We thank Nicole Pasteur for her helpful comments on the manuscript. We thank Frederic Landmann for confocal microscopy.

\section{References and recommended reading}

Papers of particular interest, published within the period of review, have been highlighted as:

- of special interest

$\bullet \bullet$ of outstanding interest

1. Flores HA, O'Neill SL: Controlling vector-borne diseases by

-• releasing modified mosquitoes. Nat Rev Microbiol 2018, 16:508-518

This review focused on new vector control methods based on the release of genetically modified or Wolbachia-infected mosquitoes.

2. Labbé P, David JP, Alout H, Milesi P, Djogbenou L, Pasteur N, Weill M: Evolution of resistance to insecticide in disease vectors. In Genetics and Evolution of Infectious Diseases, edn 2. Edited by Tibayrenc M. Elsevier Inc.; 2017:313-339.
3. Ricci I, Valzano M, Ulissi U, Epis S, Cappelli A, Favia G: Symbiotic control of mosquito borne disease. Pathog Glob Health 2012 106:380-385.

4. Werren JH, Baldo L, Clark ME: Wolbachia: master manipulators of invertebrate biology. Nat Rev Microbiol 2008, 6:741-751.

5. Zug R, Hammerstein P: Bad guys turned nice? A critical assessment of Wolbachia mutualisms in arthropod hosts. Biol Rev 2015, 90:89-111.

6. Sicard M, Dittmer J, Greve P, Bouchon D, Braquart-Varnier C: A host as an ecosystem: Wolbachia coping with environmental constraints. Environ Microbiol 2014, 16:3583-3607.

7. Laven $\mathrm{H}$ : Eradication of Culex pipiens fatigans through cytoplasmic incompatibility. Nature 1967,216:383-384

8. Dobson SL, Fox CW, Jiggins FM: The effect of Wolbachiainduced cytoplasmic incompatibility on host population size in natural and manipulated systems. Proc R Soc B Biol Sci 2002 269:437-445.

9. Zabalou S, Riegler M, Theodorakopoulou M, Stauffer C, Savakis C,

- Bourtzis K: Wolbachia-induced cytoplasmic incompatibility as a means for insect pest population control. Proc Natl Acad SCi US A 2004, 101:1 5042-15045.

The results presented in this study constituted the cornerstone to encourage methods based on the introduction of Wolbachia into pest and vector species of economic and health relevance to suppress or modify natural populations.

10. Zabalou S, Apostolaki A, Livadaras I, Franz G, Robinson AS Savakis C, Bourtzis K: Incompatible insect technique: incompatible males from a Ceratitis capitata genetic sexing strain. Entomol Exp Appl 2009, 132:232-240.

11. Hedges LM, Brownlie JC, O'Neill SL, Johnson KN: Wolbachia and Inth virus protection in insects. Science 2008, 322:702.

is studv. the 'nrotective effect' of Wolhachia adainst the Drosonhila $\mathrm{C}$ virus in Drosophila melanogaster was revealed. The infected flies showed a delay in mortality compared to flies cured of Wolbachia infection. These results indicated that Wolbachia could exhibit an antiviral effect.

12. Teixeira L, Ferreira A, Ashburner M: The bacterial symbiont

- Wolbachia induces resistance to RNA viral infections in Drosophila melanogaster. PLoS Biol 2008, 6:2753-2763.

In this study, the 'protective effects' of Wolbachia against RNA viruses (Drosophila C virus, Nora viruse and Flock House virus) and DNA virus 
(Insect Iridescent virus 6) in Drosophila melanogaster were analyzed. 'Protective effects' were found in RNA viruses but not with one DNA virus.

13. Moreira LA, Iturbe-Ormaetxe I, Jeffery JA, Lu G, Pyke AT

- Hedges LM, Rocha BC, Hall-Mendelin S, Day A, Riegler M et al.: A Wolbachia symbiont in Aedes aegypti limits infection with Dengue, Chikungunya, and Plasmodium. Cell 2009, 139:1268-1278.

This study showed that Wolbachiainfection directly inhibits the ability of a range of pathogens to infect Aedes aegypti. The effect is Wolbachia strain-specific and relates to Wolbachia priming of the mosquito innate immune system and potentially competition for limiting cellular resources required for pathogen replication.

14. WalkerT,Johnson PH, Moreira LA, Iturbe-Ormaetxe I, Frentiu FD, McMeniman CJ, Leong YS, Dong Y, AxfordJ, Kriesner Petal.: The wMel Wolbachia strain blocks dengue and invades caged Aedes aegypti populations. Nature 2011 , 476:450-455.

15. Blagrove MSC, Arias-Goeta C, Failloux A-B, Sinkins SP: Wolbachia strain wMel induces cytoplasmic incompatibility and blocks dengue transmission in Aedes albopictus. ProcNatl Acad Sci U S A 2012, 109:255-260.

16. Dutra HLC, Rocha MN, Dias FBS, Mansur SB, Caragata EP, Moreira LA: Wolbachia blocks currently circulating Zika virus isolates in Brazilian Aedes aegypti mosquitoes. Cell Host Microbe 2016, 19:771-774.

17. Aliota MT, Peinado SA, Velez ID, Osorio JE: The $u$ Mel strain of Wolbachia reduces transmission of Zika virus by Aedes aegypti. Sci Rep 2016, 6:1-7.

18. Aliota MT, Walker EC, Uribe Yepes A, Dario Velez I, Christensen BM, Osorio JE: The wMel strain of Wolbachia reduces transmission of Chikungunya virus in Aedes aegypti. PLoS Negl Trop Dis 2016, 10:e0004677.

19. Ant TH, Herd CS, Geoghegan V, Hoffmann AA, Sinkins SP: The

- Wolbachiastrain wAu provides highly efficient virus transmission blocking in Aedes aegypti. PLoS Pathog 201 8, 14:e1 006815.

In this study, the 'protective effects' of several Wolbachia strains tran sinfected in the Aedes aegypti mosquito against arboviruses (Semlik Forest, Dengue and Zika) were analyzed. Among these Wolbachia strains, the wAu strain from Drosophila simulans provided highly efficient transmission blocking of arboviruses. However, wAu did not induce $\mathrm{Cl}$ and must be associated with a Cl-inducing strain such as wAldB from Aedes albopictus to be used in vector-control strategies.

20. Frentiu FD,ZakirT, WalkerT, PopoviciJ, Pyke AT, van den HurkA, McGraw EA, O'Neill SL: Limited dengue virus replication in field-collected Aedes aegypti mosquitoes infected with Wolbachia. PLoS Negl Trop Dis 2014, 8:1-10.

21. Hoffmann AA, Montgomery BL, Popovici J, Iturbe-Ormaetxe I, Johnson PH, Muzzi F, Greenfield M, Durkan M, Leong YS, DongY et al.: Successful establishment of Wolbachia in Aedes populations to suppress dengue transmission. Nature 2011 476:454-457.

22. Hoffmann AA, Iturbe-Ormaetxe I, Callahan AG, Phillips BL, Billington K, Axford JK, Montgomery B, Turley AP, O'Neill SL: Stability of the wMel Wolbachiainfection following invasion into Aedes aegypti populations. PLoS Negl Trop Dis 2014, 8:e31 15.

23. Baldo L, Hotopp JCD, Jolley KA, Bordenstein SR, Biber SA, Choudhury RR, Hayashi C, Maiden MCJ, Tettelin H, Werren JH: Multilocus sequence typing system for the endosymbiont Wolbachia pipientis. Appl Environ Microbiol2006, 72:7098-71 10

24. Paraskevopoulos C, Bordenstein SR, Wernegreen JJ, Werren JH, Bourtzis K: Toward a Wolbachia multilocus sequence typing system: discrimination of Wolbachia strains present in Drosophila species. Curr Microbiol 2006, 53:388-395.

25. Bordenstein SR, Paraskevopoulos C, Dunning Hotopp JC, Sapountzis P, Lo N, Bandi C, Tettelin H, Werren JH, Bourtzis K: Parasitism and mutualism in Wolbachia: what the phylogenomic trees can and cannot say. Mol Biol Evol 2009, 26:231-241

26. Ros VID, Fleming VM, Feil EJ, Breeuwer JAJ: How diverse is the genus Wolbachia? Multiple-gene sequencing reveals a putatively new Wolbachia supergroup recovered from spider mites (Acari: Tetranychidae). Appl Environ Microbiol 2009, 75:1036-1043.
27. Glowska E, Dragun-Damian A, Dabert M, Gerth M: New Wolbachia supergroups detected in quill mites (Acari: Syringophilidae). Infect Genet Evol 2015, 30:140-146.

28. Turelli M, Cooper BS, Richardson KM, Ginsberg PS, Peckenpaugh B, Antelope CX, Kim KJ, May MR, Abrieux A, Wilson DA et al.: Rapid global spread of wRi-like Wolbachia across multiple Drosophila. Curr Biol 201 8, 28:963-971 .e8.

29. Jamnongluk W, Kittayapong P, Baisley KJ, O'Neill SL: Wolbachia infection and expression of cytoplasmic incompatibility in Armigeres subalbatus (Diptera: Culicidae). J Med Entomol 2000, 37:53-57.

30. Ricci I, Cancrini G, Gabrielli S, D'amelio S, Favia G: Searching for Wolbachia (Rickettsiales: Rickettsiaceae) in mosquitoes (Diptera: Culicidae): large polymerase chain reaction survey and new identifications. J Med Entomol 2002, 39:562-567.

31. Rasgon JL, Scott TW: An initial survey for Wolbachia (Rickettsiales: Rickettsiaceae) infections in selected California mosquitoes (Diptera: Culicidae): Table 1. J Med Entomol 2004, 41:255-257.

32. Baldini F, Segata N, Pompon J, Marcenac P, Robert Shaw W,

- Dabiré RK, Diabate A, Levashina EA, Catteruccia F: Evidence of natural Wolbachia infections in field populations of Anopheles gambiae. Nat Commun 2014, 5:3985.

This study suggested for the first time putative Wolbachia infections in Anopheles gambiaelong time thought as not infected with Wolbachia.

33. Shaw WR, Marcenac P, Childs LM, Buckee CO, Baldini F, Sawadogo SP, Dabire RK, Diabate A, Catteruccia F: Wolbachia infections in natural Anopheles populations affect egg laying and negatively correlate with Plasmodium development. Nat Commun 2016, 7:11772.

34. Niang EHA, Bassene H, Makoundou P, Fenollar F, Weill M, Mediannikov O: First report of natural Wolbachia infection in wild Anopheles funestus population in Senegal. Malar J 2018 , 17:1-6.

35. Baldini F, Rougé J, Kreppel K, Mkandawile G, Mapua SA, SikuluLord M, Ferguson HM, Govella N, Okumu FO: First report of natural Wolbachia infection in the malaria mosquito Anopheles arabiensis in Tanzania. Parasites Vectors 2018 , 11:635.

36. Ayala D, Akone-Ella O, Rahola N, Kengne P, Ngangue MF, MezemeF, MakangaBK, CostantiniC, SimardF, PrugnolleFetal. Natural Wolbachia infections are common in the major malaria vectors in Central Africa. bioRxiv 2018 http://dx.doi.org/10.1101/ 343715.

37. Jeffries CL, Lawrence GG, Golovko G, Kristan M, Orsborne J, Spence K, Hurn E, Bandibabone J, Tantely LM, Raharimalala FN et al.: Novel Wolbachia strains in Anopheles malaria vectors from Sub-Saharan Africa. Wellcome Open Res 2018, 3:113.

38. Chrostek E, Gerth M: Is Anopheles gambiae a natural host of - Wolbachia? bioRxiv 2018 http://dx. doi.org/10.1101/491449.

The Wolbachia infection status of Anopheles gambiae was questioned in this study as the evidences of Wolbachia infection in this species have unusual properties: high Wolbachia 16S sequence diversity, low abundance and lack of congruence between hosts. The authors believe that the unusual characteristics of Wolbachia sequences render them insufficient to diagnose Wolbachia infection and that sequence-independent evidence such as microscopic observation are required to confirm the infection.

39. Coon KL, Brown MR, Strand MR: Mosquitoes host communities of bacteria that are essential for development but vary greatly between local habitats. Mol Ecol 2016, 25:5806-5826.

40. Thongsripong $P$, Chandler JA, Green $A B$, Kittayapong $P$, Wilcox BA, Kapan DD, Bennett SN: Mosquito vector-associated microbiota: metabarcoding bacteria and eukaryotic symbionts across habitat types in Thailand endemic for dengue and other arthropod-borne diseases. Ecol Evol 2018 , 8:1352-1368.

41. Schneider DI, Riegler M, Arthofer W, Mercot H, Stauffer C, Miller WJ: Uncovering Wolbachia diversity upon artificial host transfer. PLoS One 2013, 8:e82402. 
42. Armbruster P, Damsky WE, Giordano R, Birungi J, Munstermann LE, Conn JE: Infection of new- andold-world Aedes albopictus (Diptera: Culicidae) by the intracellular parasite Wolbachia: implications for host mitochondrial DNA evolution. J Med Entomol 2003, 40:356-360.

43. Bourtzis K, Dobson SL, Xi Z, Rasgon JL, Calvitti M, Moreira LA, Bossin HC, Moretti R, Baton LA, Hughes GL et al:: Harnessing mosquito-Wolbachia symbiosis for vector and disease control. Acta Trop 2014, 132:S150-S163.

44. Zhou W, Rousset F, O'Neill S: Phylogeny and PCR-based classification of Wolbachia strains using wsp gene sequences. Proc R Soc Lond Ser B Biol Sci 1998,265:509-515.

45. Tortosa P, Charlat S, Labbe P, Dehecq J-S, Barre H, Weill M Wolbachia age-sex-specific density in Aedes albopictus: a host evolutionary response to cytoplasmic incompatibility? PLoS One 2010, 5:e9700.

46. Kambhampati S, Rai KS, Burgun SJ: Unidirectional cytoplasmic incompatibility in the mosquito, Aedes albopictus. Evolution ( $N$ Y) 1993, 47:673-677.

47. Sinkins S, Braig H, O'neill S: Wolbachia superinfections and the expression of cytoplasmic incompatibility. Proc Biol Sci 1995 261:325-330.

48. de Albuquerque AL, Magalhảes T, Ayres CFJ: High prevalence and lack of diversity of Wolbachia pipientis in Aedes albopictus populations from Northeast Brazil. Mem Inst Oswaldo Cruz 201 1, 106:773-776.

49. Atyame CM, Delsuc F, Pasteur N, Weill M, Duron O: Diversification of Wolbachia endosymbiont in the Culex pipiens mosquito. Mol Biol Evol 201 1, 28:2761-2772.

50. Dumas E, Atyame CM, Milesi P, Fonseca DM, Shaikevich EV, Unal S, Makoundou P, Weill M, Duron O: Population structure of Wolbachia and cytoplasmic introgression in a complex of mosquito species. BMC Evol Biol 2013, 13:181.

51. Atyame CM, Labbe P, Rousset F, Beji M, Makoundou P, Duron O, Dumas E, Pasteur N, Bouattour A, Fort P et al:: Stable coexistence of incompatible Wolbachia along a narrow contact zone in mosquito field populations. Mol Ecol 2015, 24:508-521.

52. Atyame CM, Pasteur N, Dumas E, Tortosa P, Tantely ML, Pocquet N, Licciardi S, Bheecarry A, Zumbo B, Weill M et al: Cytoplasmic incompatibility as a means of controlling Culex pipiens quinquefasciatus mosquito in the islands of the SouthWestern Indian Ocean. PLoS Negl Trop Dis 201 1, 5:e1440.

53. Atyame CM, Cattel J, Lebon C, Flores O, Dehecq J-S, Weill M, Gouagna LC, Tortosa P: Wolbachia-based population control strategy targeting Culex quinquefasciatus mosquitoes proves efficient under semi-field conditions. PLoS One 2015, 10:e0119288.

54. Rasgon JL, Scott TW: Wolbachia and cytoplasmic incompatibility in the California Culex pipiens mosquito species complex: parameter estimates and infection dynamics in natural populations. Genetics 2003, 165:2029-2038.

55. Dumas E, Atyame CM, Malcolm CA, Le Goff G, Unal S, Makoundou P, Pasteur N, Weill M, Duron O: Molecular data reveal a cryptic species within the Culex pipiens mosquito complex. Insect Mol Biol 2016, 25:800-809.

56. Laven H: Speciation and evolution in Culex pipiens. In Genetics of Insect Vectors of Disease. Edited by WrightJ, Pal R. Amsterdam Elsevier; 1967:251-275.

57. Atyame CM, Labbe P, Dumas E, Milesi P, Charlat S, Fort P, Weill M:

- Wolbachia divergence and the evolution of cytoplasmic incompatibility in Culex pipiens. PLoS One 2014, 9:e87336. In this study, the genetic diversity of wPip strains in the mosquito Culex pipiens and the Cl phenotypes induced by these strains were analyzed showing a significant association of Wolbachia genetic divergence and $\mathrm{Cl}$ phenotypes: most $w$ Pip strains from the same wPip group were compatible whereas those from different $w$ Pip groups were often incompatible.

58. Atyame CM, Duron O, Tortosa P, Pasteur N, Fort P, Weill M: Multiple Wolbachia determinants control the evolution of cytoplasmic incompatibilities in Culex pipiens mosquito populations. Mol Ecol 201 1, 20:286-298.

59. Yen JH, Barr AR: The etiological agent of cytoplasmic incompatibility in Culex pipiens. Jlnvertebr Pathol 1973,22:242-250.

60. Duron O, Weill M: Wolbachia infection influences the development of Culex pipiens embryo in incompatible crosses. Heredity (Edinb) 2006, 96:493-500.

61. Duron O, Fort P, Weill M: Influence of aging on cytoplasmic incompatibility, sperm modification and Wolbachia density in Culexpipiensmosquitoes. Heredity (Edinb)2007, 98:368-374.

62. Bonneau M, Landmann F, Labbe P, Justy F, Weill M, Sicard M: The

- cellular phenotype of cytoplasmic incompatibility in Culex pipiens in the light of cidB diversity. PLoS Pathog 201 8, 14 e 1007364

In this study, the cellular mechanism of $\mathrm{Cl}$ induced by Wolbachia was firs time studied in a mosquito (Culex pipiens) demonstrating similar defects than those observed in Drosophila and Nasonia.

63. McMeniman CJ, Roxanna LV, Bodil CN, Amy FWC, Manpreet S, Yu-Feng W, O'Neill SL: Stable introduction of a life-shortening Wolbachia infection into the mosquito Aedes aegypti. Science (80-) 2009, 323:141-144

64. Xi Z, Khoo CCH, Dobson SL: Wolbachia establishment and invasion in an Aedes aegypti laboratory population. Science (80) 2005, 310:326-328.

65. Fraser JE, De Bruyne JT, Iturbe-Ormaetxe I, Stepnell J, Burns RL, Flores HA, O'Neill SL: Novel Wolbachia-transinfected Aedes aegypti mosquitoes possess diverse fitness and vector competence phenotypes. PLoS Pathog 2017, 13:1-19.

66. Bian G,Joshi D, Dong Y, Lu P, Zhou G, Pan X, XuY, Dimopoulos G Xi Z: Wolbachia invades Anopheles stephensi populations and induces refractoriness to Plasmodium infection. Science (80-) $2013,340: 748-751$.

67. Blagrove MSC, Arias-Goeta C, Di Genua C, Failloux A-B, Sinkins SP: A Wolbachia wMel transinfection in Aedes albopictus is not detrimental to host fitness and inhibits Chikungunya virus. PLoS Negl Trop Dis 2013, 7:e21 52.

68. Calvitti M, Moretti R, Lampazzi E, Bellini R, Dobson SL: Characterization of a new Aedes albopictus (Diptera: Culicidae)-Wolbachia pipientis (Rickettsiales: Rickettsiaceae) symbiotic association generated by artificial transfer of the wPip strain from Culex pipiens (Diptera: Culicida). J Med Entomol 2010, 47:179-187.

69. Calvitti M, Marini F,Desiderio A, Puggioli A, Moretti R: Wolbachia density and cytoplasmic incompatibility in Aedes albopictus: concerns with using artificial Wolbachia infection as a vector suppression tool. PLoS One 2015, 10:1-19.

70. Tram U, Sullivan W: Role of delayed nuclear envelope breakdown and mitosis in Wolbachia-induced cytoplasmic incompatibility. Science 2002, 296:1124-1126.

71. Tram U, Fredrick K, Werren JH, Sullivan W: Paternal chromosome segregation during the first mitotic division determines Wolbachia-induced cytoplasmic incompatibility phenotype. J Cell Sci 2006, 119:3655-3663.

72. Landmann F, Orsi GA, Loppin B, Sullivan W: Wolbachiamediated cytoplasmic incompatibility is associated with impaired histone deposition in the male pronucleus. PLOS Pathog 2009, 5:e1000343.

73. Callaini G, Riparbelli MG, Giordano R, Dallai R: Mitotic defects associated with cytoplasmic incompatibility in Drosophila simulans. J Invertebr Pathol 1996, 67:55-64.

74. Callaini G, Dallai R, Riparbelli MG: Wolbachia-induced delay of paternal chromatin condensation does not prevent maternal chromosomes from entering anaphase in incompatible crosses of Drosophila simulans. J Cell Sci 1997, 110:271-280.

75. Beckmann JF, Bonneau M, Chen $\mathrm{H}$, Hochstrasser M, Poinsot D Mercot H, Weill M, Sicard M, Charlat S: The toxin-antidote model of cytoplasmic incompatibility: genetics and evolutionary implications. Trends Genet, in press. 
76. Beckmann JF, Fallon AM: Detection of the Wolbachia protein WPIP0282 in mosquito spermathecae: implications forcytoplasmic incompatibility. Insect Biochem Mol Biol 2013, 43:867-878.

77. Beckmann JF, Ronau JA, Hochstrasser M: A Wolbachia

- deubiquitylating enzyme induces cytoplasmic incompatibility. Nat Microbiol 2017, 2:17007.

In this paper, the capacity of the genes cidA and cidBfrom the Wolbachia wPip to induce $\mathrm{Cl}$ when transgenetically expressed in Drosophila mela nogaster was studied. $D$. melanogaster males expressing both cidA and cidBgenes were able to induce $\mathrm{Cl}$ when crossed with uninfected females, demonstrating the implication of these two genes in $\mathrm{Cl}$ induction.

78. LePage DP, Metcalf JA, Bordenstein SR, On J, Perlmutter JI,

- Shropshire JD, Layton EM, Funkhouser-Jones LJ, Beckmann JF, Bordenstein SR: Prophage WO genes recapitulate and enhance Wolbachia-induced cytoplasmic incompatibility. Nature 2017 543:243-247.

In this study, the capacity of cifAand cifBgenes from the WolbachiawMel genome to induce $\mathrm{Cl}$ when transgenetically expressed in Drosophila melanogaster was analyzed. D. melanogaster males expressing both cifAand cifBwere able to induce CI when crossed with uninfected female while males expressing either cifA or cifB genes could not. Infected temales were tertile with transgenic males showing the ability of the Wolbachia of rescuing the effect of cifA and cifB genes.

79. ShropshireJD, OnJ, Layton EM, Zhou H, Bordenstein SR: One prophage WO gene rescues cytoplasmic incompatibility in Drosophila melanogaster. Proc Natl Acad Sci U S A 2018 , 115:4987-4991.

80. Bonneau M, Atyame C, Beji M, Justy F, Cohen-Gonsaud M,

- $\quad$ Sicard M, Weill M: Culex pipiens crossing type diversity is governed by an amplified and polymorphic operon of Wolbachia. Nat Commun 2018, 9:319.

This study demonstrated the presence of several different $\operatorname{cid} A$ and $\operatorname{cid} B$ variants in each wPip strain genomes studied and the association of specific $\operatorname{cid} A$ and $\operatorname{cid} B$ variants with a given $\mathrm{Cl}$ phenotype strongly supporting the implication of these genes in the unrivaled $\mathrm{Cl}$ diversity described in Culex pipiens. 